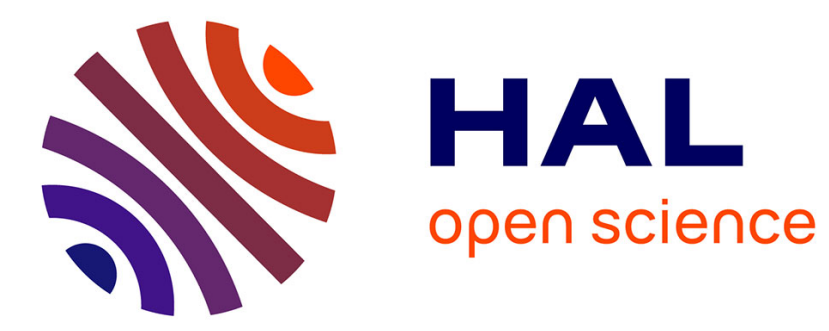

\title{
Cross-Sectional Analysis through Rank-based Dynamic Portfolios
}

\author{
Monica Billio, Ludovic Calès, Dominique Guegan
}

\section{To cite this version:}

Monica Billio, Ludovic Calès, Dominique Guegan. Cross-Sectional Analysis through Rank-based Dynamic Portfolios. 2012. halshs-00707430

\section{HAL Id: halshs-00707430 \\ https://shs.hal.science/halshs-00707430}

Submitted on 12 Jun 2012

HAL is a multi-disciplinary open access archive for the deposit and dissemination of scientific research documents, whether they are published or not. The documents may come from teaching and research institutions in France or abroad, or from public or private research centers.
L'archive ouverte pluridisciplinaire HAL, est destinée au dépôt et à la diffusion de documents scientifiques de niveau recherche, publiés ou non, émanant des établissements d'enseignement et de recherche français ou étrangers, des laboratoires publics ou privés. 


\section{Documents de Travail du Centre d'Economie de la Sorbonne}

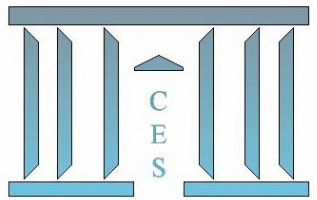

\section{Cross-Sectional Analysis through Rank-based Dynamic Portfolios}

Monica BILLIO, Ludovic CALÈS, Dominique GUEGAN

2012.36

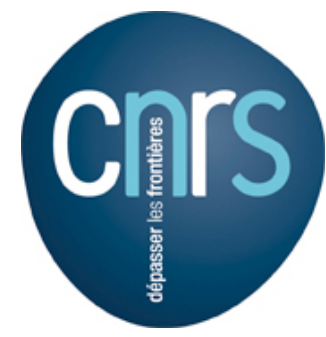




\title{
Cross-Sectional Analysis through Rank-based Dynamic Portfolios $^{\text {th }}$
}

\author{
Monica BILLIO $^{\text {a }}$, Ludovic CALÈS ${ }^{*, b, c}$, Dominique GUÉGAN ${ }^{c}$ \\ ${ }^{a}$ University Ca' Foscari of Venice - Department of Economics \\ Fondamenta San Giobbe - Cannareggio 873 - 30121 Venice, Italy \\ ${ }^{b}$ HEC - University of Lausanne - Faculty of Business and Economics \\ 1015 Lausanne, Switzerland \\ ${ }^{c}$ Paris School of Economics - University Paris-1 Panthéon-Sorbonne - MSE-CES \\ 106, avenue de l'hopital - 75013 Paris, France
}

\begin{abstract}
The aim of this paper is to study the cross-sectional effects present in the market using a new framework based on graph theory. Within this framework, we represent the evolution of a dynamic portfolio, i.e. a portfolio whose weights vary over time, as a rank-based factorial model where the predictive ability of each cross-sectional factor is described by a variable. Practically, this modeling permits us to measure the marginal and joint effects of different cross-section factors on a given dynamic portfolio. Associated to a regime switching model, we are able to identify phases during which the cross-sectional effects are present in the market.
\end{abstract}

Key words: (P) Finance, Continuous Time Random Walk, Cross-Section Analysis, Rank-based Models, Momentum JEL Classification: C31, C46, C58

\footnotetext{
${ }^{2}$ An earlier version of this paper has been presented at the Computational and Financial Econometrics conferences, in December 2010 \& 2011. We thank participants for their comments and feedback.

${ }^{*}$ Corresponding author.

Email addresses: billio@unive.it (Monica BILLIO), ludovic.cales@unil.ch (Ludovic CALÈS), dguegan@univ-paris1.fr (Dominique GUÉGAN)
} 


\section{Introduction}

The ranking of assets plays a major role in both investment decisions and empirical finance studies. Indeed, the wide-spread momentum and contrarian strategies invest according to the ranking of assets' returns, Jegadeesh and Titman (1993) and De Bondt and Thaler (1985). And the cross-section literature studies the returns of portfolios built using the rankings of assets' characteristics such as size, price-to-book value, etc. Typically, the portfolios built in these studies are zero-dollar long/short equally weighted (LSEW) portfolios where the position on an asset can only be long or short depending on the asset rank. For instance, Fama and French (1992) sort the stocks of the NYSE, Amex and Nasdaq markets according to their ratio book value to market value. Then, they form an LSEW portfolio being long the top decile and short the bottom decile. With this portfolio, they show that the top decile outperformed the bottom decile by $1.53 \%$ in average over the period 1963-1990. However, the portfolio return is a byproduct of the asset returns ranking. Indeed, the better the asset characteristics rankings explain the future assets returns ranking, the higher is the return of the portfolio. So, more information - such as the rank dependences among the assets characteristics ranking - would be retained by using the higher dimentionality of a ranking and by studying how the combination of asset characteristic rankings explain the asset returns ranking. In addition, as explained in Wärneryd (2001), people tend to think in terms of rankings rather than numbers ${ }^{1}$ when facing an investment choice. Such reasoning might have a cross-sectional effect and affect the stock returns ranking. As a consequence, a rank-based framework might be more appropriate to the study of cross-sectional effects than the conventional regression methods.

A seminal rank-based model was introduced in Mallows (1957). Considering a set of true rankings $\pi$ and a set of explanatory rankings $\sigma$, it estimates the degree of expertise of $\sigma$ in explaining $\pi$ as the dispersion factor of the distribution describing the distance between $\pi$ and $\sigma$. This model is provided in its

\footnotetext{
1"If people assign probabilities to future states, they are likely to think in terms of ranks, that is, one outcome is held to be more likely than another, and they do not use precise probabilities or even probability intervals. A major problem is that there may be a correlation between prefered state and rank, since people tend to overestimate the likelihood of prefered outcomes.", Wärneryd (2001)
} 
modern version by Diaconis (1988) and generalized to multiple explanatory rankings by Fligner and Verducci (1986) and Lafferty and Lebanon (2002). Its applications are mostly found in the field of machine learning, e.g. Fligner and Verducci (1986), Lafferty and Lebanon (2002), Busse et al. (2007) and Cheng and Hüllermeier (2009). As suggested in Diaconis (1988), the results of Billio et al. (2011a) stem from graph theory and provide an explicit formulation of Mallows' model. This formulation leads to a rank-based framework used to study the momentum effect. Considering the set of portfolios defined by the LSEW investment strategy, they build a graph where the nodes are the portfolios and the edges correspond to a minimal change between two portfolios. In this framework, each portfolio corresponds to a possible ranking. Then, defining a dynamic portfolio as a sequence of portfolios over a rolling window, i.e. a portfolio whose weights vary over time, they model its dynamics as a stochastic process on the graph. A dynamic portfolio of particular interest is the optimal dynamic portfolio which is the sequence of the portfolios providing the highest returns over each of the rolling windows. Each of these optimal portfolios corresponds to a ranking of the assets returns over one period. The dynamics of the optimal portfolio is modeled as an autoregressive process where the steps between two optimal portfolios are due to a random walk. Then, the number of random steps determines the presence of momentum effect. If the number of random steps is low enough, two successive optimal portfolios are likely to be close and the momentum effect is observed. Otherwise, investing in the optimal portfolio would yield no significant returns and there would be no momentum effect. Remark that this framework assumes that all the asset returns have the same dynamics and makes no assumption about market efficiency. In addition, the risk taken by investing in a LSEW portfolio is mostly captured by the number of permutations between the invested portfolio and the optimal portfolio, see Fig.3 in Billio et al. (2011a). In the related area of performance measure, we refer to Billio et al. (2011b). At last, the dynamics of the optimal portfolio might not be only driven by an autoregressive process. As seen in the previous paragraph, the cross-section literature has identified several cross-sectional factors affecting the optimal portfolio. The aim of this paper is to extend the rank-based framework developed in Billio et al. (2011a), and to use it for the development of models dedicated to the study of cross-sectional effects in financial markets.

In this paper, we first extend the discrete step random walk model introduced 
in Billio et al. (2011a) to continuous time in order to get the probability for the dynamic portfolio following a random walk to be at a given location on the graph after a given period of time instead of a number of steps. It allows us to work with the average number of steps walked by the dynamic portfolio. Coupled with a Markov switching model, we use it empirically in order to identify periods of momentum effect in the U.S. market. Secondly, we introduce univariate models taking into account the influence of factors on the optimal portfolio. This is achieved considering that a part of the path walked by the optimal portfolio is explained by a factor, i.e. a portfolio built from the ranking of assets characteristics. Then, the unexplained part of the path - from the factor portfolio to the optimal portfolio - is supposed to follow a random walk. It permits us to study empirically the cross-section effects introduced by different factors such as the price-to-book value ratio and the dividend yields in several markets. Thirdly, we propose multivariate models - taking into consideration the factors' possible dependences - for the dynamics of the optimal portfolio. These models are multivariate convolution models where each explanatory ranking is the origin of a random walk leading to the explained ranking. The models proposed here are bivariate combining the effects of two factors among market value, price earning and dividend yield - and trivariate - combining the effects of market value, price earning and dividend yield. The marginal and joint cross-section effects of these factors are then studied in several markets.

The paper is divided as follows. Section 2 presents the framework. First, we extend Billio et al. (2011a) by modeling the dynamics of the optimal portfolio with a continuous time random walk model. Secondly, we propose univariate models where a cross-sectional factor drives the dynamics of the optimal dynamic portfolio. Thirdly, we extend the previous models to the multivariate case allowing the study of the marginal and joint cross-section effects of several factors. Section 3 presents applications of the previous framework. First, we study the momentum effect. Secondly, we study the cross-sectional effects of different company characteristics in several markets. Thirdly, we study the marginal and joint cross-section effects of several factors. Section 4 concludes. 


\section{The Framework}

\subsection{From Discrete Step to Continuous Time}

The LSEW strategy consists in investing in portfolios which are long/short (including both long and short positions), zero-dollar (the value of the long positions is equal to the value of the short positions) and equally weighted (each position has the same value in absolute value). It is usual to consider that the leverage of these portfolios is $2: 1^{2}$ which is the leverage used in the following. This strategy is commonly used to track the momentum effect in most of the literature [Jegadeesh and Titman (1993), Rouwenhorst (1998), Chan et al. (2000), Okunev and White (2003), Kazemi et al. (2009) and Billio et al. (2011a) among others].

An elegant representation of this strategy is provided by a graph theoretic approach where each node represents a portfolio and each edge corresponds to a single change in position between portfolios. The distance $h$ between two portfolios is the minimum number of permutations to pass from one to the other, i.e. it is the Cayley distance [Cayley (1859)]. We denote $\Gamma$ the set of the investable portfolios $\gamma$ induced by this strategy. In a market of $n$ assets, we represent a portfolio as a weight vector, i.e. $\gamma=(\gamma(1), \ldots, \gamma(n))^{\prime}$ where $\gamma(i)$ is the weight associated with asset $i, i=1, \ldots, n$, and $\gamma^{\prime}$ is the transpose of $\gamma$. As shown in Billio et al. (2011a), the graph representation of this strategy has interesting properties: it is 2-antipodal and distance regular. From the 2-antipodal property we can compute the eigenvalues of the adjacency matrix associated with the graph, and the distance regular property leads to Theorem 1 below. Before to reach the main theorem, we need to introduce the stratification of $\Gamma$ following Hora and Obata (2007). Considering a given portfolio $\gamma^{*} \in \Gamma$, the disjoint union of strata $\Gamma_{n}$ such that

$$
\Gamma=\bigcup_{n=0}^{\infty} \Gamma_{n}, \Gamma_{n}=\left\{\gamma \in \Gamma \mid h\left(\gamma, \gamma^{*}\right)=n\right\}
$$

is the stratification of $\Gamma$ associated with the portfolio $\gamma^{*}$, given by the minimum number of permutations which separate $\gamma^{*}$ from the portfolios of each

\footnotetext{
${ }^{2}$ The notation 2:1 means that the amount of capital backing the portfolio represents $50 \%$ of the portfolio value. It is the minimum amount required under the U.S. Regulation (namely Regulation T). As a consequence, the sum of the absolute values of the weights of the portfolio equals 2 .
} 
stratum.

Now, we consider a dynamic portfolio - defined as a sequence of portfolios - following a random walk on the graph $G=(\Gamma, E)$. Thus, considering a rolling window $\left(T_{1}, \ldots, T_{n}\right)$ where each $T_{i}$ has the same length $T$, we assume that $h\left(\gamma_{T_{i}}, \gamma_{T_{i+1}}\right) \leq 1, \forall i \in\{1, \ldots, n\}$ and each move has the same probability of occurrence. In the case of the LSEW momentum strategy, this means that two consecutive portfolios differ by at most one permutation. ${ }^{3}$ This hypothesis is relevant for a dynamic portfolio based on returns as soon as we expect that two consecutive portfolios with overlapping periods are very close. We can note that the choice of a long period and a short frequency makes this assumption particularly relevant.

The probability for a dynamic portfolio following a random walk on $G$ to be at a distance $n$ from its origin portfolio after $N$ random steps has been established in Billio et al. (2011a) and we recall it now:

Theorem 1. If $G$ is a finite distance-regular graph with diameter $d$ and intersection table

$$
\left(\begin{array}{ccccc}
c_{0} & c_{1} & c_{2} & \ldots & c_{d} \\
a_{0} & a_{1} & a_{2} & \ldots & a_{d} \\
b_{0} & b_{1} & b_{2} & \ldots & b_{d}
\end{array}\right)
$$

then the probability for dynamic portfolio $\gamma_{N}$, following a random walk on $G$, to be at a distance $n$ from the original portfolio $\gamma_{o}$ after $N$ random moves on $G$ is :

$$
P\left(h\left(\gamma_{N}, \gamma_{o}\right)=n\right)=\frac{\sqrt{\left|\Gamma_{n}\right|}}{d^{2 N}\left\|P_{n}\right\||\Gamma|} \sum_{i=0}^{d} m_{i} \lambda_{i}^{N} P_{n}\left(\lambda_{i}\right)
$$

where $\lambda_{i}$ are the eigenvalues of the adjacency matrix of the graph $G$, with their multiplicities $m_{i}$, and $\left\{P_{i}(x)\right\}_{i=0}^{d}$ are the Gram-Schmidt polynomials, defined as below in the three-term recurrence relation:

\footnotetext{
${ }^{3}$ We can remark that $T_{i}$ and $T_{i+1}, i \in\{1, \ldots, n\}$, are overlapping.
} 


$$
\begin{aligned}
& P_{0}(x)=1 \\
& P_{1}(x)=x-a_{0} \\
& x P_{i}(x)=P_{i+1}(x)+a_{i} P_{i}(x)+b_{i-1} c_{i} P_{i-1}(x), i=1,2, \ldots
\end{aligned}
$$

In our case, the graph $\Gamma$ is such that $a_{n}=2 n(d-n), b_{n}=(d-n)^{2}$ and $c_{n}=n^{2}$. However, the larger the number of of assets, the higher is the degree of $P_{n}$. It follows that in practice, this method is limited to around $N=20$ assets. A future extension of the paper is to approximate $\frac{\sqrt{\left|\Gamma_{n}\right|}}{d^{2 N}|\Gamma|} P_{n}\left(\lambda_{i}\right)$ for larger $N$.

The previous random walk can also be represented as a continuous-time Markov chain where each state corresponds to a portfolio and the probabilities to pass from a portfolio to any neighbor portfolio are identical. In this uniform continuous-time Markov chain, the waiting time in any state follows an exponential distribution with parameter $\nu t$, where $\nu \in \mathbb{R}$ is given for a unit time. Thus, the random variable $N(t)$ of number of jumps over a period of time $t$ is a Poisson distribution with parameter $\nu t$. The expected number of steps over a period of length 1 is $\nu$ and we have

$$
P[N(t)=k]=\frac{e^{\nu t}(\nu t)^{k}}{k !}
$$

Thus, the probability for a dynamic portfolio to be at a distance $\mathrm{n}$ from its origin after a period of time $t$ is given by:

$$
P\left[h\left(\gamma_{t}, \gamma_{o}\right)=n, \nu\right]=\frac{\sqrt{\left|\Gamma_{n}\right|}}{\left\|P_{n}\right\||\Gamma|} \sum_{i=0}^{d} m_{i} P_{n}\left(\lambda_{i}\right) e^{-\nu t} e^{\frac{\lambda_{i} \nu t}{d^{2}}} .
$$

Using the Taylor expansion of the exponential function $\sum_{k=0}^{\infty} \frac{\lambda_{i}^{k}(\nu t)^{k}}{d^{2 k} k !}=e^{\frac{\lambda_{i} \nu t}{d^{2}}}$, we get:

$$
P\left[h\left(\gamma_{t}, \gamma_{o}\right)=n, \nu\right]=\frac{\sqrt{\left|\Gamma_{n}\right|}}{\left\|P_{n}\right\||\Gamma|} \sum_{i=0}^{d} m_{i} P_{n}\left(\lambda_{i}\right) e^{\left(\frac{\lambda_{i}}{d^{2}}-1\right) \nu t}
$$

So, the probability for a dynamic portfolio to be at a distance $n$ from its origin after a period of time $t$ is given by relationship (7). Intuitively, the lower is $\nu$, the fewer are the number of random steps and the closer are $\gamma_{o}$ and $\gamma_{t}$. In Section 3.1, we use expression (7) in order to estimate the number 
of random steps wandered by a dynamic portfolio following a random walk and to study the momentum effect.

\subsection{Univariate Models for Dynamic Portfolios}

In the previous section, we assumed that the distances between two successive optimal portfolios were independent and identically distributed. In order to be more flexible, we assume that some dependence exists and that it can be captured by a factor.

Let consider two dynamic portfolios $\gamma_{X}$ and $\gamma_{Y}$ represented as sequences of portfolios observed at time $t, t \in\{1, \ldots, T\}: \gamma_{X}=\left\{\gamma_{X, t=1}, \gamma_{X, t=2}, \ldots\right\}$ and $\gamma_{Y}=\left\{\gamma_{Y, t=1}, \gamma_{Y, t=2}, \ldots\right\}$. Let assume that the dynamics of $\gamma_{Y}$ is partly explained by $\gamma_{X}$. Then, we model the dynamics of $\gamma_{Y}$ between the times $t$ and $t+1$ as the composition of a path from $\gamma_{Y, t}$ to $\gamma_{X, t}$, which is explained by factor $X$, and a path from $\gamma_{X, t}$ to $\gamma_{Y, t+1}$ which is random. Such a modeling is possible because the steps of $\gamma_{Y}$ are a composition of permutations and thus they can be rewritten as a composition of two sets of permutations. Noting $\sigma_{Y}$ the path from $\gamma_{Y, t}$ to $\gamma_{Y, t+1}$ and $\sigma_{X}$ the path from $\gamma_{Y, t}$ to $\gamma_{X, t}$, the idea is to re-write $\sigma_{Y}$ as the composition of $\sigma_{X}$ and a random path from $\gamma_{X, t}$ to $\gamma_{Y, t+1}$ denoted $\sigma_{\epsilon}: \sigma_{Y}=\sigma_{\epsilon} \circ \sigma_{X}$. This composition is illustrated in Figure 1.

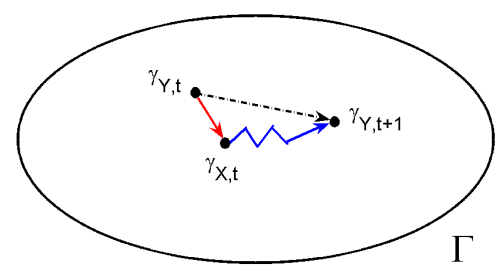

Figure 1: Composition of the walk from $\gamma_{Y, t}$ to $\gamma_{Y, t+1}$ as a determined walk from $\gamma_{Y, t}$ to $\gamma_{X, t}$ and a random walk from $\gamma_{X, t}$ to $\gamma_{Y, t+1}$.

The paths $\sigma_{X}$ and $\sigma_{\epsilon}$ do not need to be identified as the aim of our modeling is to infer the number of steps walked by $\sigma_{\epsilon}$. Observing the two sequences of portfolios $\gamma_{Y}$ and $\gamma_{X}$, we can compute the sequence of the distances between $\gamma_{X}$ and $\gamma_{Y}$ denoted $\left(h_{X, Y}\right)_{t}=\left\{h_{X, Y}(t)=h\left(\gamma_{X, t}, \gamma_{Y, t+1}\right)\right\}_{t=1}^{T-1}$ in a sample of size $T$. Because the walk $\sigma_{\epsilon}$ is unexplained, theses distances are assumed to be due to a random walk, i.e. to be distributed according to the following relationship: 


$$
P\left[h_{X, Y}(t)=n, \nu\right]=\frac{\sqrt{\left|\Gamma_{n}\right|}}{\left\|P_{n}\right\||\Gamma|} \sum_{i=0}^{d} m_{i} P_{n}\left(\lambda_{i}\right) e^{\left(\frac{\lambda_{i}}{d^{2}}-1\right) \nu}
$$

Intuitively, the lower is $\nu$, the fewer are the random steps and the better $\gamma_{X}$ explains $\gamma_{Y}$, over the given sample. In Section 3.2, we use the relationship (8) to study some cross-sectional effects in the U.S. and European markets.

\subsection{Multivariate Models of Portfolio Dynamics: The Case of Independent Factors}

In order to extend the results of the previous section, we want to take into account the effect of several factors on a dynamic portfolio. In this section, we assume independent factors.

Consider the dynamic portfolio $\gamma_{Y}$ explained by $m$ factors denoted $X_{1}, \ldots, X_{m}$. We denote $h_{X_{i}, Y}(t)$ the distance between the portfolios $\gamma_{X_{i}}$ at date $t$ and $\gamma_{Y}$ at date $t+1$. In the univariate case, the number of random steps associated with a factor $X$ can be estimated ${ }^{4}$ assuming that the difference between $\gamma_{Y, t+1}$ and the factor portfolio $\gamma_{X, t}$ is due to a random walk and using the distribution (8). Now, the model being multivariate, each random walk issued from a portfolio $\gamma_{X_{i}, t}$ has to lead $\gamma_{Y, t+1}$, i.e. the $m$ random walks have to meet in $\gamma_{Y, t+1}$. So, we need to extend the distribution (8) conditionally to the fact that the walk of each factor $\gamma_{X_{i}, t}$ leads to $\gamma_{Y, t+1}$. In this section, we assume that each random walk leads to $\gamma_{Y, t+1}$ independently of each other. In other words, the random walks do not share any common step. The bivariate case is illustrated in Figure 2.

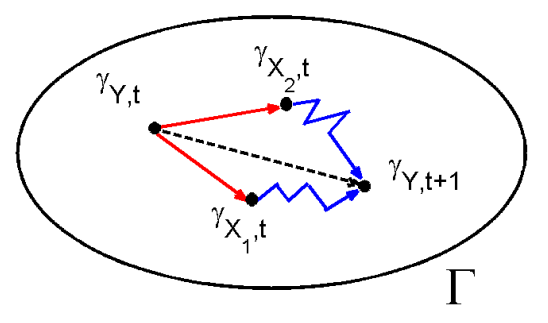

Figure 2: Random walks of two independent variables.

\footnotetext{
${ }^{4}$ see Section 3.2.
} 
Remarking that independent random walks may not lead to the same portfolio and in order to provide the multivariate extension of the distribution (8), we first compute the unconditional probability that the $m$ random walks lead to a portfolio $\gamma \in \Gamma$ which is at given distances from the $m$ origins $\left(\gamma_{X_{i}}\right)_{i=1}^{m}$. Then, we compute the unconditional probability that the $m$ random walks meet in any portfolio $\gamma \in \Gamma$, i.e. the sum of the previous probabilities. Finally, we provide the probability for the $m$ random walks to meet at certain distances from their origins $\left(\gamma_{X_{i}}\right)_{i=1}^{m}$ conditionally to the fact that they lead to the same portfolio.

First, remark that the probability for a dynamic portfolio to be in any portfolio of a given stratum is the same for all the portfolios of this stratum. So, the probability for a dynamic portfolio starting in $\gamma_{o}$ to be at a specific portfolio $\gamma_{t} \in \Gamma_{n}$ after a period of time $t$ is

$$
P\left[\gamma_{o}=\gamma_{t}, \nu\right]=\frac{P\left[h\left(\gamma_{o}, \gamma_{t}\right)=n, \nu\right]}{\left|\Gamma_{n}\right|}, n \in\{0, \ldots, d\} \text { and } \gamma_{t} \in \Gamma_{n}
$$

If the factors $\left(\gamma_{X_{i}}\right)_{i=1}^{m}$ are the origins of $m$ random walks, we get several stratifications of $\Gamma$. Such stratifications are illustrated in Figure 3 in the bivariate case.

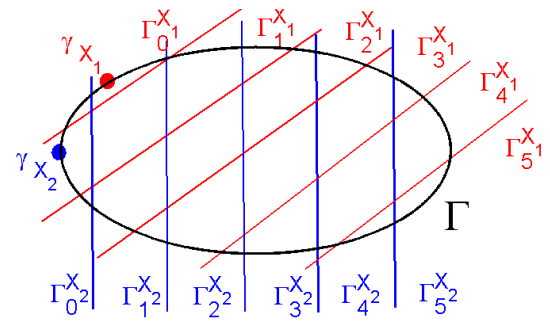

Figure 3: Stratification with two origins $\gamma_{X_{1}}$ and $\gamma_{X_{2}}$ at a distance $n_{1,2}=1$ from each other.

So, in a graph of diameter $d$, the number of portfolios $\gamma \in \Gamma$ at the given distances $\left\{n_{i}\right\}_{i=1}^{m} \in\{0, \ldots, d\}^{m}$ from the portfolios $\gamma_{X_{i}}$ is

$$
\left|\bigcap_{i=1}^{m} \Gamma_{n_{i}}^{X_{i}}\right|
$$

This number of portfolios (10) depends on the distances between the factors $\left(\gamma_{X_{i}}\right)_{i=1}^{m}$. In the bivariate case, the number of portfolios at a distance $n_{1}$ 
from $X_{1}$ and $n_{2}$ from $X_{2}$ depends on the distance $n_{1,2}$ between $X_{1}$ and $X_{2}$. The LSEW graph being distance regular, this number of portfolios can be computed, Billio et al. (2011a). For instance, in a market of 10 assets, the number of portfolios at distances 0 to 5 from two factor portfolios which are themselves at a distance $n_{1,2}=1$ from each other, is illustrated in Table 1 below.

\begin{tabular}{|c|cccccc|}
\hline$n_{1} \backslash n_{2}$ & 0 & 1 & 2 & 3 & 4 & 5 \\
\hline 0 & 0 & 1 & 0 & 0 & 0 & 0 \\
1 & 1 & 8 & 16 & 0 & 0 & 0 \\
2 & 0 & 16 & 48 & 36 & 0 & 0 \\
3 & 0 & 0 & 36 & 48 & 16 & 0 \\
4 & 0 & 0 & 0 & 16 & 8 & 1 \\
5 & 0 & 0 & 0 & 0 & 1 & 0 \\
\hline
\end{tabular}

Table 1: Number of portfolios $\gamma \in \Gamma$, i.e. $p_{n_{1}, n_{2}}^{n_{1,2}}$, at the distances $n_{1}$ from $\gamma_{X_{1}}$ and $n_{2}$ from $\gamma_{X_{2}}$ when $n_{1,2}=1$.

Assuming that each random walk originating in $\gamma_{X_{i}}, i=\{1, \ldots, m\}$ leads to $\gamma_{Y} \in \Gamma$ independently, the probability for the $m$ random walks to meet in a portfolio $\gamma_{Y}$ of sub-stratum $\bigcap_{i=1}^{m} \Gamma_{n_{i}}^{X_{i}}$ is

$$
P\left[h_{X_{i}, Y}(t)=n_{i}, \nu_{i} ; i=1, \ldots, m\right]=\left|\bigcap_{i=1}^{m} \Gamma_{n_{i}}^{X_{i}}\right| \prod_{i=1}^{m} \frac{P\left[h_{X_{i}, Y}(t)=n_{i}, \nu_{i}\right]}{\left|\Gamma_{n_{i}}\right|}
$$

So, the probability that the $m$ random walks meet in any portfolio $\gamma_{Y} \in \Gamma$ is

$$
\sum_{n_{1}=0}^{d} \cdots \sum_{n_{m}=0}^{d}\left|\bigcap_{i=1}^{m} \Gamma_{n_{i}}^{X_{i}}\right| \prod_{i=1}^{m} \frac{P\left[h_{X_{i}, Y}(t)=n_{i}, \nu_{i}\right]}{\left|\Gamma_{n_{i}}\right|}
$$

where $d$ is the diameter of the graph.

Finally, the conditional probability that the $m$ random walks meet in substratum $\bigcap_{i=1}^{m} \Gamma_{n_{i}}^{X_{i}}$ after a period of time $t$, conditionally to the fact that they lead to the same portfolio $\gamma_{Y}$ is given by the ratio of (11) over (12): 


$$
P\left[h_{X_{i}, Y}(t)=n_{i}, \nu_{i} ; i=1, \ldots, m\right]=\frac{\left|\bigcap_{i=1}^{m} \Gamma_{n_{i}}^{X_{i}}\right| \prod_{i=1}^{m} \frac{P\left[h_{X_{i}, Y}(t)=n_{i}, \nu_{i}\right]}{\left|\Gamma_{n_{i}}\right|}}{\sum_{n_{1}=0}^{d} \ldots \sum_{n_{m}=0}^{d}\left|\bigcap_{i=1}^{m} \Gamma_{n_{i}}^{X_{i}}\right| \prod_{i=1}^{m} \frac{P\left[h_{X_{i}, Y}(t)=n_{i}, \nu_{i}\right]}{\left|\Gamma_{n_{i}}\right|}}
$$

In Section 3.3, these multivariate models are used to study cross-sectional effects in the U.S. markets.

\subsection{Multivariate Models of Portfolio Dynamics: The Case of Dependent Factors}

As in the previous section, we consider the dynamic portfolio $\gamma_{Y}$ explained by $m$ factors noted $X_{1}, \ldots, X_{m}$. We note $h_{X_{i}, Y}(t)$ the distance between the portfolios $\gamma_{X_{i}}$ at date $t$ and $\gamma_{Y}$ at date $t+1$. Here, we assume that the factors are dependent in such a way that the $m$ random walks meet before to share some steps leading to $\gamma_{Y}$. An illustration of this modeling, in the bivariate case, is provided below in Figure 4.

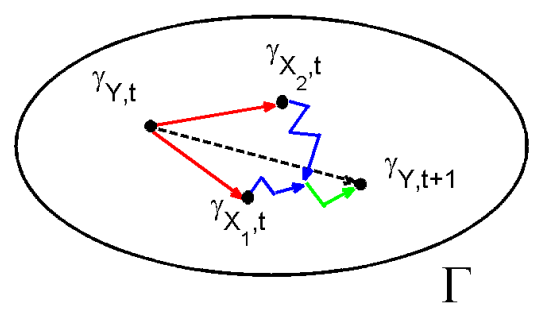

Figure 4: Random walks of two dependent variables.

For the sake of simplicity, the main part of the following section focuses on the bivariate case. Considering two processes $X_{1}$ and $X_{2}$ with parameters $\nu_{1}$ and $\nu_{2}$, they are decomposed into a common process with parameter $\nu_{1,2}$ and two idiosyncratic processes with parameters $\nu_{1}^{\prime}$ and $\nu_{2}^{\prime}$. The dependence between $X_{1}$ and $X_{2}$ is quantified by the following correlation-like coefficient, Ruan et al. (2008) :

$$
\rho_{1,2}=\frac{\nu_{1,2}}{\nu_{1,2}+\nu_{1}^{\prime}+\nu_{2}^{\prime}}
$$

Intuitively, two processes behaving identically share all their steps leading to a coefficient equal to one. On the contrary, two processes sharing none of 
their steps lead to a coefficient equal to zero, and thus $0 \leq \rho_{1,2} \leq 1$.

In the case of $m$ factors, we can consider different structures. As an illustration, possible structures for the dependences in the trivariate case are reported in Figure 5. Structure 1 illustrates a common dependence between three factors. Structure 2 illustrates a common dependence between three factors with an additional dependence between two factors.

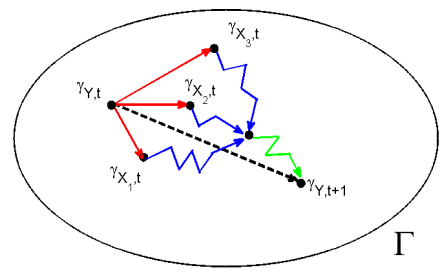

Structure 1

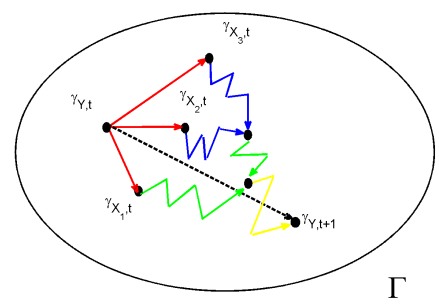

Structure 2

Figure 5: Dependence structures of random walks from three dependent variables.

Back to the bivariate case, we assume that two random walks originating from two factor portfolios have the non-null probability to meet at certain distances from their origins, given by equation (13). These random walks are characterized by their parameters $\nu_{1}^{\prime}$ and $\nu_{2}^{\prime}$. Once the two random walks have met, they both follow a single random walk which is characterized by a single parameter $\nu_{1,2}$. It is illustrated in Figure 4. So, the resulting probability to find $\gamma_{Y}$ at given distances from the origins portfolios is the convolution of the probability of the random walks to meet in a portfolio $\gamma_{i} \in \Gamma$ and the probability for a random walk beginning in $\gamma_{i}$ and with parameter $\nu_{12}$ to end in $\gamma_{Y} \in \Gamma$. This probability is given by :

$$
\begin{aligned}
& P\left[\gamma_{Y}=\gamma,\left(\nu_{1}, \nu_{2}, \nu_{1,2}\right) ; h_{X_{1}, X_{2}}=n_{1,2}, \gamma \in \Gamma\right] \\
= & \sum_{\gamma_{i} \in \Gamma} \frac{P\left[h_{X_{1}, \gamma_{i}}=n_{1}, h_{X_{2}, \gamma_{i}}=n_{2},\left(\nu_{1}^{\prime}, \nu_{2}^{\prime}\right) ; h_{X_{1}, X_{2}}=n_{1,2}\right]}{\left|\Gamma_{n_{1}} \cap \Gamma_{n_{2}}\right|} \times \frac{P\left[h_{\gamma_{i}, \gamma}=n, \nu_{1,2}\right]}{\left|\Gamma_{n}\right|}
\end{aligned}
$$

In Section 3.4, these multivariate models are used to study joint crosssectional effects in the U.S. market. 


\section{Applications}

\subsection{Application to the study of the Momentum Effect}

In this section, we use the relationship (7) developed in Section 2.1 to study the momentum effect in the U.S. and European markets. These markets are constituted by the ten sectoral indexes provided by Datastream. ${ }^{5}$ The data are daily and range from $01 / 01 / 1973$ to $02 / 08 / 2010$. In the same way as in Billio et al. (2011a), we study the momentum effect through the dynamics of the optimal dynamic portfolio which is the sequence of portfolios, among the LSEW portfolios, providing the highest returns over successive periods. In the case of LSEW portfolios, the optimal portfolio is long the half best performers and short the half worst performers of each period. Here, the optimal portfolio is built each day using the daily returns of the assets. We note $\gamma_{t}$ the optimal portfolio built using the return of the assets on day $t$. Next, we assume that the optimal dynamic portfolio follows the random walk described in the first section. So, the steps of the optimal portfolio between $t$ and $t+1$ are random and the probability for $\gamma_{t+1}$ to be at a distance $n$ from $\gamma_{t}$ is given by equation (7).

Intuitively, the lower is $\nu$ the closer are two successive optimal portfolios and the stronger is the momentum effect. Given the sequence of optimal portfolios $\left(\gamma_{1}, \ldots, \gamma_{T}\right)$ where $\mathrm{T}$ is the sample size, we build the sequence $(h(t))_{t=1}^{T-1}$ of the distances between two successive optimal portfolios such that $h(t)=h\left(\gamma_{t}, \gamma_{t+1}\right)$, for $t=1$ to $T$. Assuming that the sequence $(h(t))_{t=1}^{T-1}$ is independent and identically distributed, we compute $\hat{\nu}$ using the maximum likelihood method with the following log-likelihood function:

$$
\ell(\hat{\nu})=\sum_{t=1}^{T-1} \log \left(\frac{\sqrt{\left|\Gamma_{h(t)}\right|}}{\| P_{h(t)}|||\Gamma|} \sum_{i=0}^{d} m_{i} P_{h(t)}\left(\lambda_{i}\right) e^{\left(\frac{\lambda_{i}}{d^{2}}-1\right) \hat{\nu}}\right)
$$

Thus, using $\hat{\nu}$ given by relationship (16) we get an estimate of the distribution

\footnotetext{
${ }^{5}$ Datastream codes:

- for the U.S.market : OILGSUS, BMATRUS, INDUSUS, CNSMGUS, HLTHCUS, CNSMSUS, TELCMUS, UTILSUS,FINANUS and TECNOUS

- for the European market : OILGSEU, BMATREU, INDUSEU, CNSMGEU, HLTHCEU, CNSMSEU, TELCMEU, UTILSEU, FINANEU and TECNOEU
} 
of the distances between two consecutive portfolios. When $\nu$ is very large, we have ${ }^{6}$

$$
\lim _{\nu \rightarrow \infty} P[h(t)=n, \nu]=\frac{\left|\Gamma_{n}\right|}{|\Gamma|}
$$

Relationship (17) implies that the distribution of the distances is symmetric, i.e. that we have the same probability to find the dynamic portfolio at any location on the graph. So, the origin portfolio provides no information on the final location of the dynamic portfolio and no momentum effect is observed.

To detect the presence of momentum effect, we test whether the distribution (7) implied by $\hat{\nu}$ is symmetric or not through a $\chi^{2}$ goodness-of-fit test between this estimated distribution and the symmetric one. In Table 2 we report the estimated number of random steps and the p-value of the associated $\chi^{2}$ goodness-of-fit test ${ }^{7}$ for the U.S. and European markets.

\begin{tabular}{|l|c|c|}
\hline Cross-Sectional Factor & U.S. Market & European Market \\
\hline Est. Nber of Random Steps $: \hat{\nu}$ & 6.75 & 6.50 \\
$\chi^{2}$ G.o.F. (p-value) & 0.00 & 0.00 \\
\hline
\end{tabular}

Table 2: This table reports the estimated number of random steps $\hat{\nu}$ (the lower the better), and the p-value of the associated $\chi^{2}$ goodness-of-fit test, of the optimal dynamic portfolio in the U.S. and European markets. The markets are constituted by the ten sectoral indexes provided by Datastream. The data are daily and range from 01/01/1973 to 02/08/2010.

The estimated number of daily random steps is 6.5 in the European market and 6.75 in the U.S. market, so the momentum effect is significantly present in both samples. In addition, both numbers of random steps are significant as indicated by the p-values of the $\chi^{2}$ test which are very small.

Until now we have modeled the dynamics of the optimal dynamic portfolio assuming that the number of random steps is constant over the whole sample. In order to take into account the possibility of phases in the momentum effect we relax this last assumption and allow the number of random steps to take different values. The underlying idea is that different market activity would

\footnotetext{
${ }^{6}$ Proof available upon request to the authors.

${ }^{7}$ Remark that, in a sample of 9500 observations of 10 assets, this test is significant at the $1 \%$ level for a number of random steps lower than 10.8 .
} 
impact differently the number of random steps. For instance, one can expect that if more information disturbs the market, the optimal dynamic portfolio would walk more random steps. So, by letting $\nu$ take two values, $\nu_{1}$ and $\nu_{2}$, we get two different regimes for the walk of the dynamic portfolio leading to two different distributions of its distance to the origin. These two distributions are as follows:

$$
\left\{\begin{array}{l}
P\left[h(t)=n \mid \nu=\nu_{1}\right]=\frac{\sqrt{\left|\Gamma_{k}\right|}}{\left\|P_{k}\right\||\Gamma|} \sum_{i=0}^{d} m_{i} e^{\left(\frac{\lambda_{i}}{d^{2}}-1\right) \nu_{1}} P_{k}\left(\lambda_{i}\right) \\
P\left[h(t)=n \mid \nu=\nu_{2}\right]=\frac{\sqrt{\left|\Gamma_{k}\right|}}{\left\|P_{k}\right\||\Gamma|} \sum_{i=0}^{d} m_{i} e^{\left(\frac{\lambda_{i}}{d^{2}}-1\right) \nu_{2}} P_{k}\left(\lambda_{i}\right)
\end{array}\right.
$$

We suppose that the transitions between these two regimes can be modeled with a Markov switching model Hamilton (1989) which identifies the two regimes of the momentum effect. Under the same assumption used previously, we get estimates for $\nu_{1}$ and $\nu_{2}$ by the maximum likelihood method. ${ }^{8}$ Their values are presented in Table 3 .

\begin{tabular}{|l|cc|}
\hline & $\hat{\nu}$ & $\chi^{2}$ G.o.F. (p-value) \\
\hline State 1 & 5.75 & 0.00 \\
State 2 & $1.26 \times 10^{8}$ & 1 \\
\hline
\end{tabular}

Table 3: This table reports the estimated numbers of random steps (the lower the better), and the p-value of the associated $\chi^{2}$ goodness-of-fit, of the optimal dynamic portfolio in the U.S. market of the ten sectoral indexes provided by Datastream from 01/01/1973 to 02/08/2010. The data are daily.

We obtain a small value for $\hat{\nu}_{1}$ and a large value for $\hat{\nu}_{2}$. The associated pvalues suggest that the distribution obtained with $\hat{\nu}_{1}$ is not symmetric while the distribution obtained with $\hat{\nu}_{2}$ is symmetric. So, state 1 is identified as the momentum regime and state 2 corresponds to a regime with no momentum effect. We illustrate the discrete distribution of the distances between two consecutive optimal portfolios for each state in Figure 6.

The smoothed probabilities resulting from the Markov switching model are provided in Figure 7. These probabilities correspond to the a posteriori probabilities, i.e. with the knowledge of the whole sample, to be in the regime

\footnotetext{
${ }^{8}$ Here, there is no closed-form likelihood function because we follow Hamilton (1989) were the likelihood function is the result of an iterative algorithm.
} 


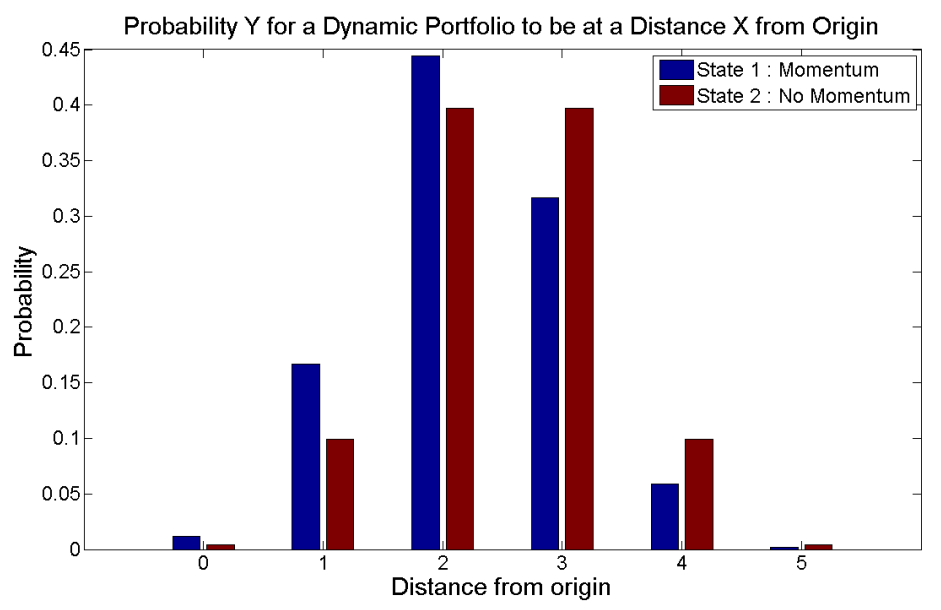

Figure 6: Distributions associated with each regime. The distribution corresponding to the momentum effect is skewed toward 0 and corresponds to the blue bars. The distribution of the regime with no momentum is symmetric and is represented with the red bars.

of momentum. From the beginning of the sample to 1997, the momentum effect was a permanent effect, with only three periods of absence (77-79, 80, 87-89). From 1997, the momentum effect is recurrent but it is not as stable as it was before, lasting only few months. It is in line with the results of Hwang and Rubesam (2008) who suggested that the momentum effect disappeared in the late 1990's. The statistics on the returns of the momentum strategy for each regime are provided in Table 4. As expected, these returns are significantly positive during the momentum regime and not significantly different from zero otherwise.

\begin{tabular}{|l|cc|}
\hline & Momentum Regime & No Momentum Regime \\
\hline Avg. Return & $0.14 \%$ & $0.01 \%$ \\
T-stat & 23.19 & 1.25 \\
\hline
\end{tabular}

Table 4: This table reports the average returns and their associated $t$-statistics of the momentum strategy for the momentum regime and the regime without momentum effect. These regimes are those identified in the previous paragraph with the smoothed probabilities. The data is daily and range from 1973 to 2010.

We used the same model with the European market. However, no phase of momentum effect could be found. It suggests that the momentum effect is more diffuse in this market. 


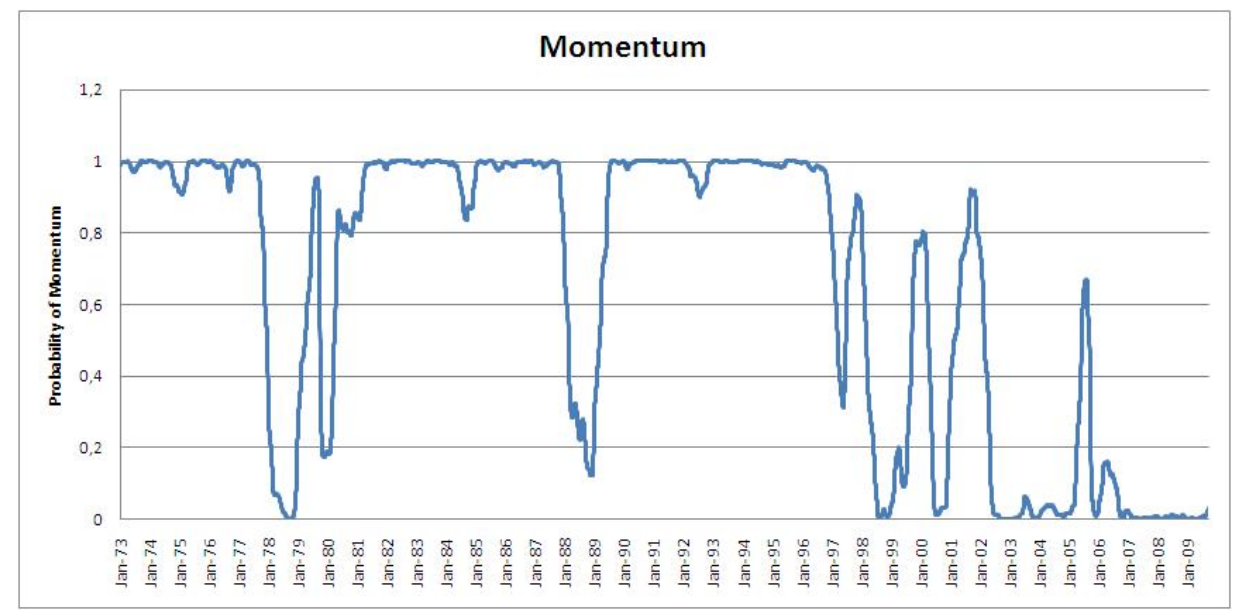

Figure 7: Smoothed probabilities to be in a phase of Momentum effect in the U.S. market.

\subsection{Applications to the study of cross-sectional effects}

In this section, we use distribution (8) developed in Section 2.2 to study single cross-sectional effects in several markets. Considering the U.S., European and Asian markets constituted by the ten sectoral indexes provided by Datastream, we aim to explain the dynamics of the optimal dynamic portfolio, $\gamma_{Y}$, using as factors the market value, the price earning or the dividend yield of the assets. Considering the asset characteristic $X$, we build the factor portfolio $\gamma_{X}$ as follows. First, we rank the assets from the highest value of $\mathrm{X}$ to its lowest value. Next, we split the set of assets in two halves, assigning a long position to the assets with the highest values of $\mathrm{X}$ and a short position to the rest of them. However, a cross-sectional effects may result from the opposite ranking of a factor (being long the lowest values and short the highest). So, we also consider the inverse of a factor portfolio $\gamma_{X}$, i.e. $-\gamma_{X}$. For instance, in the application on the U.S. market, we consider the inverse dividend yield which is long the assets with the lowest dividend yield and short the assets with the highest dividend yield. The use of the inverse dividend yield in the U.S. might be counter-intuitive because of the positive relationship between dividend yield and returns usually found in the literature at the firm level. However, at the sectoral level, Günsel and Çukur (2007) show the presence of the inverse dividend yield effect in the U.K. market. Here, we are observing the same effect for the U.S. market.

Assuming that $\left(h_{X, Y}\right)_{t}$ is a sequence of independent and identically dis- 
tributed, we compute the estimate number of random steps $\hat{\nu}$ distancing $\gamma_{Y}$ from $\gamma_{X}$ by maximizing the following log-likelihood function over the whole sample:

$$
\ell(\hat{\nu})=\log \prod_{t=1}^{T} P\left[h_{X, Y}(t), \hat{\nu}\right]
$$

In Table 5, we provide the estimations $\bar{\nu}$ obtained with the previous factors in the U.S. market. As a benchmark, we also report the result obtained for momentum, i.e. using the previous optimal portfolio as factor. In this sample, the momentum effect has the lowest $\hat{\nu}(\hat{\nu}=7.16)$ so it is the strongest cross-section effect. It is followed by the inverse dividend yield $(\hat{\nu}=8.16)$ which is significant and by the market value $(\hat{\nu}=8.69)$ and price earning $(\hat{\nu}$ $=9.14$ ) which are not significant as indicated by their high p-values.

We report the estimations of the number of random steps $\hat{\nu}$ for the European and Asian markets in Table 6. In the literature, both positive and negative relationships between the assets' returns and their sizes, price earnings and dividend yields can be found. In particular, Claessens et al. (1995) and Singh (2008) find that the signs of size and dividend yield effects on stocks returns are opposite in emerging markets to those of developed markets. The reasons for these opposite relationships remains unclear. As a consequence, if the factors considered for the U.S. market provide no cross-sectional effects for the European and Asian markets, we use the inverse factors. In the European market, the momentum is the strongest effect $(\hat{\nu}=7.88)$ and the only one to be significant. In the Asian market, the strongest effect is provided by the dividend yield $(\hat{\nu}=7.28)$ followed by the momentum effect $(\hat{\nu}=7.68)$.

Next, following the previous section, we estimate the Markov switching models with the inverse dividend yield, for the U.S. market, and the dividend yield, for the Asian market. In the U.S. market, we were unable to identify any phase. It suggests that this cross-section effect is aphasic in this market. On the contrary, in the Asian market, we identify a phase of dividend yield effect. The estimations of the coefficients are provided in Table 7 and the smoothed probabilities to be in the phase of dividend yield effect are provided in Figure 8. We identify three phases of dividend yield effect: the first one 


\begin{tabular}{|l|cc|}
\hline Cross-Sectional Factor & \multicolumn{2}{|c|}{ U.S. Market } \\
& $\hat{\nu}$ & $\chi^{2}$ G.o.F. (p-value) \\
\hline Momentum & 7.16 & 0.001 \\
Market Value & 8.69 & 0.143 \\
Price Earning & 9.14 & 0.260 \\
Inverse Dividend Yield & 8.16 & 0.051 \\
\hline
\end{tabular}

Table 5: This table reports the estimated numbers of random steps (the lower the better), and the p-value of the associated $\chi^{2}$ goodness-of-fit, of the optimal dynamic portfolio when using as factors the momentum, the market value, the inverse dividend yield and the price earning. The results presented are for the U.S. market constituted by the ten sectoral indexes provided by Datastream. The data are monthly and range from 01/01/1973 to $02 / 08 / 2010$, i.e. 452 observations. Remark that with a sample of 452 observations, the $\chi^{2}$ goodness-of-fit test is significant at the $10 \%$ level for a number of steps lower than 8.48.

\begin{tabular}{|l|cc|cc|}
\hline Cross-Sectional Factor & \multicolumn{2}{|c|}{ European Market } & \multicolumn{2}{c|}{ Asian Market } \\
& $\hat{\nu}$ & $\chi^{2}$ G.o.F. $(\mathrm{p}$-value) & $\hat{\nu}$ & $\chi^{2}$ G.o.F. (p-value) \\
\hline Momentum & 7.88 & 0.024 & 7.68 & 0.012 \\
Inverse Market Value & 9.47 & 0.355 & 9.15 & 0.261 \\
Inverse Price Earning & 13.19 & 0.949 & 10.07 & 0.527 \\
Dividend Yield & 8.93 & 0.202 & 7.28 & 0.002 \\
\hline
\end{tabular}

Table 6: This table reports the estimated numbers of random steps (the lower the better), and the p-value of the associated $\chi^{2}$ goodness-of-fit, of the optimal dynamic portfolio when using as factors the momentum, the market value, the inverse dividend yield and the price earning. The results presented are for the European and Asian markets constituted by the ten sectoral indexes provided by Datastream. The data are monthly and range from $01 / 01 / 1973$ to $02 / 08 / 2010$, i.e. 452 observations. Remark that with a sample of 452 observations, the $\chi^{2}$ goodness-of-fit test is significant at the $10 \%$ level for a number of steps lower than 8.48 .

from the beginning of the sample to April 1977, the second one from March 1984 to July 1995 and the third one from February 2000 to June 2009.

\begin{tabular}{|l|cc|}
\hline & $\hat{\nu}$ & $\chi^{2}$ G.o.F. (p-value) \\
\hline State 1 & 5.53 & 0.00 \\
State 2 & 36.49 & 1 \\
\hline
\end{tabular}

Table 7: This table reports the estimated numbers of random steps (the lower the better), and the p-value of the associated $\chi^{2}$ goodness-of-fit, walked by the random walk issued from the dividend yield portfolio and leading to the optimal portfolio in the Asian market of the ten sectoral indexes provided by Datastream from 31/01/1973 to 29/10/2010. The data are monthly. 


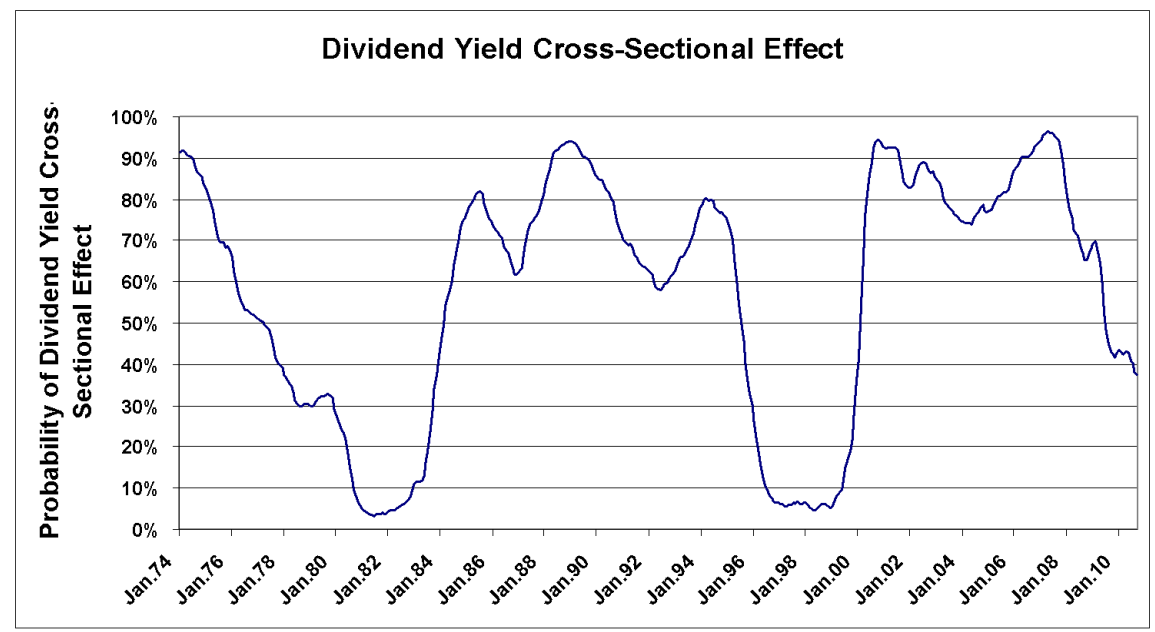

Figure 8: Smoothed probabilities to be in a phase of Dividend Yield effect in the Asian market.

\subsection{Applications of multivariate models to the study of independent cross- sectional effects}

In this section, we use distribution (13) developed in Section 2.3 to jointly study independent cross-sectional effects in the U.S. market made of the 10 sectoral Datastream indexes. The factors are the price earning (PE), the market value (MV) and the inverse dividend yield (iDY) and the explained variable is the optimal portfolio. We estimate the parameters $\left(\nu_{i}\right)_{i=1}^{m}$ in (13) by maximum likelihood following the same methodology as presented in the previous section. However, the estimated numbers of random steps associated with each factor are different from those computed independently from Equation (19). ${ }^{9}$ Indeed, the multivariate model takes into account the new information of the distance between the factors. The log-likelihood function is

$$
\ell\left(\hat{\nu}_{1}, \ldots, \hat{\nu}_{m}\right)=\log \prod_{t=1}^{T-1}\left(\frac{\left|\bigcap_{i=1}^{m} \Gamma_{n_{i}}^{X_{i}}\right| \prod_{i=1}^{m} \frac{P\left[h_{X_{i}, Y}(t)=n_{i}, \nu_{i}\right]}{\left|\Gamma_{n_{i}}\right|}}{\sum_{n_{1}=0}^{d} \ldots \sum_{n_{m}}^{d}\left|\bigcap_{i=1}^{m} \Gamma_{n_{i}}^{X_{i}}\right| \prod_{i=1}^{m} \frac{P\left[h_{X_{i}, Y}(t)=n_{i}, \nu_{i}\right]}{\left|\Gamma_{n_{i}}\right|}}\right)
$$

\footnotetext{
${ }^{9}$ This is an extension of the classical machine learning literature, Lafferty and Lebanon (2002).
} 
where the parameters $\nu_{i}$ correspond to the predictive ability of factor $X_{i}$. Intuitively, the lower is $\nu_{i}$ the greater is the predictive ability of $X_{i}$.

Using (20), we estimate the predictive ability of these factors $\left(\hat{\nu}_{P E}, \hat{\nu}_{M V}\right.$ and $\left.\hat{\nu}_{i D Y}\right)$ by combining them in bi- and tri-variate models. The results are presented in Table 8 . The results of the univariate models come from Table 5. They are computed using (19) and are reported as benchmarks.

\begin{tabular}{|c|c|c|c|}
\hline Univariate Models & $\hat{\nu}_{P E}$ & $\hat{\nu}_{M V}$ & $\hat{\nu}_{i D Y}$ \\
\hline Model $1(X=\mathrm{PE})$ from $(19)$ & 9.14 & & \\
\hline Model $2(X=\mathrm{MV})$ from $(19)$ & \multicolumn{3}{|c|}{8.69} \\
\hline Model $3(X=\mathrm{iDY})$ from $(19)$ & & & 8.14 \\
\hline Bivariate Models & $\hat{\nu}_{P E}$ & $\hat{\nu}_{M V}$ & $\hat{\nu}_{i D Y}$ \\
\hline Model $4\left(X_{1}=\mathrm{PE}, X_{2}=\mathrm{MV}\right)$ from $(20)$ & 10.36 & 9.22 & \\
\hline Model $5\left(X_{1}=\mathrm{MV}, X_{2}=\mathrm{iDY}\right)$ from $(20)$ & & 10.55 & 8.82 \\
\hline Model $6\left(X_{1}=\mathrm{PE}, X_{2}=\mathrm{iDY}\right)$ from $(20)$ & 13.23 & & 8.35 \\
\hline Trivariate Model & $\hat{\nu}_{P E}$ & $\hat{\nu}_{M V}$ & $\hat{\nu}_{i D Y}$ \\
\hline Model $7\left(X_{1}=\mathrm{PE}, X_{2}=\mathrm{MV}, X_{3}=\mathrm{iDY}\right)$ from $(20)$ & 14.10 & 10.59 & 8.99 \\
\hline
\end{tabular}

Table 8: Estimations of the number of random steps walked by the random walks issued from the lagged price earning factor $\left(\hat{\nu}_{P E}\right)$, the lagged market value factor $\left(\hat{\nu}_{M V}\right)$ and the lagged inverse dividend yield $\left(\hat{\nu}_{i D Y}\right)$ to the optimal portfolio considering uni-, bi- and trivariate models, in the U.S. market composed by the 10 sectoral Datastream indexes. The data are monthly (end-of-month quotations) and range from 31/01/1973 to 29/10/2010.

The results of the bivariate models confirm the results of the univariate models suggesting that the predominant effect is the inverse dividend yield effect. Indeed it provides the lowest number of random steps among those of the models 4,5 and 6 . The same result is observed in the trivariate model ${ }^{10}$ model 7 - where the inverse dividend yield factor provides the lowest number of random steps.

\subsection{Applications of multivariate models to the study of dependent cross- sectional effects}

In this section, we use distribution (15) developed in Section 2.4 to study dependent cross-sectional effects in the U.S. market made of the 10 sectoral

\footnotetext{
${ }^{10}$ In the trivariate case, the number of portfolios in the sub-stratum has been computed by enumeration.
} 
Datastream indexes. Consider the bivariate models using two of the following lagged factors: price earning (PE), market value (MV) and inverse dividend yield (iDY), which explain the optimal portfolio. We estimate the idiosyncratic and shared number of random steps of the random walks originating from each factor portfolio in the same way as in the previous sections. Using equation (15) and considering the factors $X_{1}$ and $X_{2}$ explaining the portfolio $Y$, the $\log$-likelihood function used to estimate $\nu_{1}^{\prime}, \nu_{2}^{\prime}$ and $\nu_{1,2}$ is

$$
\ell\left(\nu_{1}^{\prime}, \nu_{2}^{\prime}, \nu_{1,2}\right)=\log \prod_{t=1}^{T-1} P\left[\gamma_{Y},\left(\nu_{1}, \nu_{2}, \nu_{1,2}\right) ; h_{X_{1}, X_{2}}=n_{1,2}\right]
$$

The estimation of the idiosyncratic average number of steps for each factor, denoted $\hat{\nu}_{P E}^{\prime}, \hat{\nu}_{M V}^{\prime}$ and $\hat{\nu}_{P E}^{\prime}$, as well as their average number of shared random steps, denoted $\hat{\nu}_{\text {Common }}$, are computed using equation (21). The dependence between the random walks issued from the two factors of each model is quantified by the coefficient $\rho_{c}$ defined in equation (14). The results are presented below in Table 9 .

\begin{tabular}{|l|cccc|c|}
\hline Bivariate Models & $\hat{\nu}_{P E}^{\prime}$ & $\hat{\nu}_{M V}^{\prime}$ & $\hat{\nu}_{i D Y}^{\prime}$ & $\hat{\nu}_{C o m m o n}$ & $\rho_{c}$ \\
\hline Model $8\left(X_{1}=\mathrm{PE}, X_{2}=\mathrm{MV}\right)$ from $(21)$ & 7.35 & 6.27 & & 2.96 & 0.18 \\
Model $9\left(X_{1}=\mathrm{MV}, X_{2}=\mathrm{iDY}\right)$ from $(21)$ & & 5.38 & 3.87 & 5.02 & 0.35 \\
Model $10\left(X_{1}=\mathrm{PE}, X_{2}=\mathrm{iDY}\right)$ from $(21)$ & 9.24 & & 5.18 & 3.26 & 0.18 \\
\hline
\end{tabular}

Table 9: Estimations of the number of random steps walked by the random walks issued from the lagged price earning factor $\left(\hat{\nu}_{P E}^{\prime}\right)$, the lagged market value factor $\left(\hat{\nu}_{M V}^{\prime}\right)$ and the lagged inverse dividend yield $\left(\hat{\nu}_{i D Y}^{\prime}\right)$ to the optimal portfolio considering a bivariate models taking into account the dependence between the factors. The estimation of the average number of shared steps between two factors is denoted $\hat{\nu}_{C o m m o n}$. The dependence between the random walks issued from the two factors of each model is quantified by $\rho_{c}$ introduced in equation 14. The market considered is the U.S. market composed by the 10 sectoral Datastream indexes. The data are monthly (end-of-month quotations) and range from $31 / 01 / 1973$ to $29 / 10 / 2010$

The number of random steps estimated in the independent case, from (20), and in the dependent case, from (21), are in line with each other as shown in Table 10. Indeed, we have the following relationships:

$$
\begin{aligned}
& \hat{\nu}_{P E} \approx \hat{\nu}_{P E}^{\prime}+\hat{\nu}_{C o m m o n} \\
& \hat{\nu}_{M V} \approx \hat{\nu}_{M V}^{\prime}+\hat{\nu}_{C o m m o n} \\
& \hat{\nu}_{i D Y} \approx \hat{\nu}_{i D Y}^{\prime}+\hat{\nu}_{C o m m o n}
\end{aligned}
$$




\begin{tabular}{|l|ccc|}
\hline Bivariate Models from $(20)$ & $\left(\hat{\nu}_{P E}\right)$ & $\left(\hat{\nu}_{M V}\right)$ & $\left(\hat{\nu}_{i D Y}\right)$ \\
\hline Model $4\left(X_{1}=\mathrm{PE}, X_{2}=\mathrm{MV}\right)$ & 10.36 & 9.22 & \\
Model $5\left(X_{1}=\mathrm{MV}, X_{2}=\mathrm{iDY}\right)$ & & 10.55 & 8.82 \\
Model 6 $\left(X_{1}=\mathrm{PE}, X_{2}=\mathrm{iDY}\right)$ & 13.23 & & 8.35 \\
\hline Bivariate Models from $(21)$ & $\left(\hat{\nu}_{P E}^{\prime}+\hat{\nu}_{\text {Common }}\right)$ & $\left(\hat{\nu}_{M V}^{\prime}+\hat{\nu}_{\text {Common }}\right)$ & $\left(\hat{\nu}_{i D Y}^{\prime}+\hat{\nu}_{\text {Common }}\right)$ \\
\hline Model $8\left(X_{1}=\mathrm{PE}, X_{2}=\mathrm{MV}\right)$ & 10.31 & 9.23 & \\
Model $9\left(X_{1}=\mathrm{MV}, X_{2}=\mathrm{iDY}\right)$ & & 10.40 & 8.89 \\
Model $10\left(X_{1}=\mathrm{PE}, X_{2}=\mathrm{iDY}\right)$ & 12.50 & & 8.44 \\
\hline
\end{tabular}

Table 10: This table presents the number of random steps estimated in the independent case, obtained from Table 8 , and the sum of the idiosyncratic and joint numbers of random steps obtained in Table 9 in the U.S. market composed by the 10 sectoral Datastream indexes. The data are monthly (end-of-month quotations) and range from 31/01/1973 to 29/10/2010. The factors considered are the lagged price earning (PE), the lagged market value $(\mathrm{MV})$ and the lagged inverse dividend yield. In the independent case, the associated estimated number of random steps are denoted respectively $\hat{\nu}_{P E}, \hat{\nu}_{M V}$ and $\hat{\nu}_{i D Y}$. In the dependent case, the associated estimated idiosyncratic number of random steps are denoted respectively $\hat{\nu}_{P E}^{\prime}, \hat{\nu}_{M V}^{\prime}$ and $\hat{\nu}_{i D Y}^{\prime}$, and the estimated number of shared random steps is denoted $\hat{\nu}_{\text {Common }}$.

The dependence between the random walks has for main effect to give higher probabilities to become the next optimal portfolio to the distant portfolios. Indeed, in the dependent case, the random walks meet after fewer random steps than in the independent case. So, the condition that they have to meet in a portfolio before the final portfolio is less restrictive than the assumption to meet in the final portfolio for which they pursue their way with the common random walk.

\section{Conclusion}

In this paper, we propose new models for the dynamics of the optimal portfolio in the case of the LSEW strategy. In a first part, we extend the discrete step random walk model introduced in Billio et al. (2011a) to continuous time. By this way the number of random steps does not have to be determined and is defined in terms of probability. This modeling is then coupled with a Markov switching model in order to empirically identify periods of daily momentum in the U.S. market. The results suggest that the momentum effect disappeared in the late 90's.

Next, we introduce the influence of factors on the optimal dynamic portfolio. 
In a first part, we consider the effect of a single factor. It allows to measure the cross-sectional effects of several single factors. The results suggest that the strongest effects are those induced by the previous asset returns (momentum) and by the dividend yields of the assets. In a second part, we consider the effects of several factors. It enables us to consider the marginal and joint effects of these factors. As an application, we measure the dependency among the different factors (price earning, market value and dividend yield) in the U.S. market. These measures suggest that the most correlated factors are the market value and the dividend yield.

This modeling provides the probability for any portfolio of the graph to be the next optimal portfolio. Thus, a direct application would concern its use in asset management. In particular, we can propose to estimate the idiosyncratic and joint abilities of analysts ranking assets.

\section{References}

Billio, M., Calès, L., \& Guégan, D. (2011). Portfolio Symmetry and Momentum. European Journal of Operational Research, 214, 759-767.

Billio, M., Calès, L., \& Guégan, D. (2011). A Cross-Sectional Score for the Relative Performance of an Allocation. International Review of Applied Financial Issues and Economics, 3(4), 700-710

Busse L., Orbanz P. and Buhmann J. (2007). Cluster Analysis of Heterogeneous Rank Data, Proceeding of the 24th Internatinal Conference on Machine Learning, 113-120

Cayley, A. (1859). A Sixth Memoir upon Quantics. Philosophical Transactions Royal Society, 149, 61-90.

Chan, K., Alaudeen, H., \& Wilson, T. (2000). Profitability of momentum strategies in the international equity markets. Journal of Financial and Quantitative Analysis, 35, 153-172.

Cheng W. and Hüllermeier E. (2009). A New Instance-Based Label Ranking Approach Using the Mallows Model, Advances in Neural Networks, LNCS 5551, 707-716 
Claessens, S., Dasgupta, S., \& Glen, J. (1995). The Cross-Section of Stock Returns: Evidence from Emerging Markets, World Bank Papers, 1995

De Bondt, W., \& Thaler, R. (1985). Does the stock market overreact ? Journal of Finance, 40, 793-805.

Diaconis, M. (1988). Group representations in probability and statistics, Institute of Mathematical Statistics, 1988

Fama, E., \& French, K. (1992). The Cross-Section of Expected Stock Returns. Journal of Finance, 47(2), 427-465.

Fligner, M. \& Verducci J. (1988). Distance Based Ranking Models, Journal of the Royal Statistical Society Series B (Methodological), 48(3), 359-369

Günsel, N., \& Çukur, S. (2007). The Effects of Macroeconomic Factors on the London Stock Returns: A Sectoral Approach. International Research Journal of Finance and Economics, 10, 140-152.

Hora, A., \& Obata, N. (2007). Quantum Probability and Spectral Analysis of Graphs. Springer-Verlag.

Hamilton, J. (1989). A New Approach to Economic Analysis of Nonstationary Time Series and the Business Cycle. Econometrica, 57, 357-384.

Hwang, S., \& Rubesam, A. The Disappearance of Momentum. Working Paper.

Jegadeesh, N., \& Titman, S. (1993). Returns to buying winners and selling losers: Implications for stock market efficiency. Journal of Finance, 48, $65-91$.

Kazemi, H., Schneeweis, T., \& Spurgin, R. (2009). Momentum in asset returns: are commodity returns a special case ? Journal of Alternative Investments, 10, 23-36.

Lafferty, J., \& Lebanon, G. (2002). Cranking: Combining Rankings using Conditional Probability Models on Permutations. Proceedings of the Nineteenth International Conference on Machine Learning, 363-370

Mallows C. (1957). Non-null Ranking Models, Biometrika, 44, 114-130 
Okunev, K., \& White, D. (2003). Do momentum-based strategies still work in foreign currency markets? Journal of Financial and Quantitative Analysis, 38, 425-447.

Rouwenhorst, K. (1998). International Momentum Strategies. Journal of Finance, 53, 267-284.

Ruan, S., MacEachern, S., Otter, T., \& Dean, A. (2008). The dependent Poisson race model and modeling dependence in conjoint choice experiments. Psychometrika, 73(2), 261-288.

Singh, R. (2008). Company attributes and Cross-Section of stock returns in India, Asia-Pacific Business Review, 2008

Wärneryd, K-E. (2001). Stock Market Psychology: How People Value and Trade Stocks, Edward Elgar Publishing, 2001 